\title{
Physical Chemistry of Dust: Weak Intermolecular Interactions in Molecular Nanoparticles
}

\author{
Ruth Signorell* \\ Werner Prize Winner 2005
}

\begin{abstract}
Large weakly bound molecular aggregates are ubiquitous: As atmospheric aerosols they influence the quality of the air and have a significant impact on our climate. Furthermore pharmaceuticals in the form of nanoparticles are required to achieve a targeted drug delivery. In all these fields, physical-chemical methods play a crucial role for the preparation and characterization of this type of matter. Apart from such experimental efforts, one of the greatest challenges is to understand the properties of large molecular aggregates on a microscopic level.
\end{abstract}

Keywords: Collisional cooling · Infrared spectroscopy · Intermolecular interactions · Molecular nanoparticles · Rapid expansion of supercritical solutions

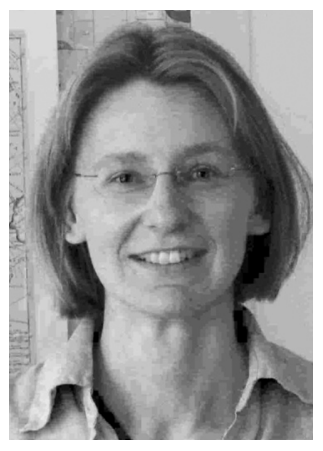

Ruth Signorell, born 1969 in Thusis, studied Interdisziplinäre Naturwissenschaften at ETH Zürich. She received her diploma in 1996 with the thesis 'Ferninfrarotspektroskopie von $\mathrm{HFDF}$ und $\mathrm{CH}_{2} \mathrm{D}_{2}$ : Zentrifugalverzerrungseffekte auf Linienpositionen und Intensitäten'. Working on high-resolution photoelectron spectroscopy with Prof. Frédéric Merkt at ETH Zürich she received

\footnotetext{
${ }^{*}$ Correspondence: Prof. Dr. R. Signorell

Department of Chemistry

University of British Columbia

2036 Main Mall

Vancouver, B.C., V6T 1 Z1

Canada

Tel.: +1 6048229064

Fax: +1 6048222847

E-Mail: signorell@chem.ubc.ca
}

her Dr. sc. nat. in 1999 with a thesis on 'Struktur und Dynamik von Molekülkationen'. Ruth Signorell was postdoctoral fellow of the Deutsche Akademie der Naturforscher LEOPOLDINA (2000-01) and later on Wissenschaftliche Assistentin $\mathrm{C} 1$ (2001-02) at the Georg-August University Göttingen, where she worked with Prof. Martin Suhm on infrared spectroscopy of molecular aggregates. From 2002 to 2005 Ruth Signorell was Assistant Professor for Physical Chemistry at the University of Göttingen. Since 2005 she is Professor for Physical and Analytical Chemistry at the University of British Columbia in Vancouver. Current research interests are centred around the generation and spectroscopic characterization of molecular nanoparticles and aerosols in the submicron size range.

\section{Introduction}

The rapidly increasing interest in the properties of clusters, nanoparticles, and aerosols built from molecules arises from their relevance in many different fields. Molecular particles of widely varying composition have a significant influence on atmospheric processes [1]. At the same time, small particles in the form of atmospheric aerosols can pose a risk to our health. Solid 'ice' particles containing water, carbon monoxide, carbon dioxide, and various small organic molecules also exist in interstellar dust, where their properties and their influence, for example as reactive sites, are still largely unexplored [2]. The subject of another very active field is the targeted preparation of micro- and nanoparticles of pharmaceutical agents [3]. The micronization of pharmaceuticals improves their bioavailability, and in combination with surface modification this can be exploited for a targeted and enhanced drug uptake.

With sizes ranging from subnanometers to microns, molecular particles typically contain from some tens up to billions of molecules. Common to all these species is the fact that they are held together by rather weak intermolecular forces (Van der Waals forces, hydrogen bonds) acting between the different molecules. These weak forces contrast with the strong chemical bonds within the molecules themselves. This pronounced hierarchy of forces in combination with the huge change in size make this type of matter particularly complicated to investigate and understand. It is thus not astonishing that many properties are still largely unexplored. However, for the targeted preparation of particles and the modeling of processes involving particles reference data are urgently needed, and these can only be obtained from corresponding laboratory investigations.

The hierarchy of forces mentioned has also a crucial influence on the particles properties. In the investigation of particulate matter intrinsic particle properties such as shape and size effects are of particular interest, because both the shape and the size of the 
particles can have a characteristic influence on the phase behavior, the reactivity, and the spectroscopic properties. The present contribution discusses some of these phenomena and their microscopic origins.

\section{Experimental Approaches}

The study of particulate matter requires the combination of a wide range of different experimental techniques both for the particle preparation and for the particle characterization. A major difficulty is the generation of submicron particles in the lab with welldefined size distributions and that for different substances. For volatile substances, we use two methods, collisional cooling [4-9] and supersonic expansion [10][11]. In both cases particles are formed directly from the gas phase by bringing a dilute sample gas to supersaturation. In collisional cooling cells this is realized by injecting the sample gas into a precooled bath gas (helium, $\mathrm{T}>4 \mathrm{~K}$ ) and in supersonic expansions the particle formation is induced by rapid expansion of the sample gas through a small nozzle. The combination of both methods allows us to generate particles from subnanometers to several microns, which is a prerequisite to study size-dependent effects. With collisional cooling we can also study the temporal behavior of an aerosol over several hours as well as investigate phase transitions and reactions. Conventional particle preparation methods for non-volatile substances, such as milling or spray drying, are not suitable to form submicron particles. Such particles can rather be generated by rapid expansion of supercritical solutions (RESS) [12][13] or by electrospraying [14]. The key idea behind RESS is to dissolve the solute of interest in a supercritical medium (i.e. $\mathrm{CO}_{2}$ ) and to precipitate this solution by rapid expansion through a small nozzle. In an electrospray the particles are formed by spraying an aqueous solution with subsequent solvent evaporation. The latter technique only works for compounds that are soluble in polar solvents.

For the characterization of molecular nanoparticles infrared spectroscopy is particularly well suited. It opens a direct window to the characteristic intermolecular and intramolecular vibrational dynamics of these species. Furthermore it is sensitive, non-invasive, and can be used for various kinds of particulate samples under a broad range of different experimental conditions. The usage of specially designed in situ techniques [12][15] enables us, for example, to observe the particle formation in the aerosol phase and to probe the properties of particles in supersonic expansions. We complement the results from in situ infrared spectroscopy with particle sizing using a scanning mobility particle sizer, three-wavelengths-extinction measurements, and electron microscopy. The latter also provides information about the particle's shape. As further off-line techniques we also use X-ray diffraction and differential scanning calorimetry [16].

\section{Infrared Patterns of Shape and Size}

In recent years we have systematically studied the infrared signatures of very weakly bound molecular aggregates, which are stable only under cryogenic conditions [4][5][10][11][17][18]. This includes solid particles consisting of water, carbon dioxide, ammonia, sulfur dioxide, and various composite particles. In the lab, we generate these particles in collisional cooling cells as aerosols and study them in situ by infrared spectroscopy. These 'ice' particles are of relevance in the atmosphere and in the interstellar dust. They are also important for the generation of non-volatile particles by rapid expansion of supercritical solutions (see Section 5) where the solvent forms ice particles during the expansion process [12]. One of the first questions that comes to mind in this context is whether the finite size leads to any characteristic features in infrared spectra.

\subsection{Shape Effects}

Fig. 1 illustrates that this is indeed the case [4][17][18]. The left-hand side shows experimental and calculated (see Eqn. (1)) spectra of pure $\mathrm{CO}_{2}$ particles in the region of the antisymmetric stretching vibration, for two different particle shapes, in panel a) for predominantly elongated particles, and in panel b) for mostly spherical particles. The comparison of the two panels clearly reveals the strong influence the particle shape has on the structure of the infrared absorption band. An elongated particle leads to a broad band with three different maxima whilst a spherical particle is characterized essentially by a single narrow absorption. This example demonstrates that it is possible to draw conclusions on the particle shape directly from infrared spectra.

A central point for the understanding of the microscopic origin of these phenomena is the prediction of infrared spectra based on quantum mechanical models. However, for the large aggregates considered here, which contain about $10^{6}$ molecules (diameter $\sim 50 \mathrm{~nm}$ ), it is not possible to perform such calculations ab initio having regard to all intra- and intermolecular interactions. For the species studied here, we have shown that this is not really required [19] because in this case a single intermolecular interaction is responsible for the shape sensitivity. It is the resonant coupling between the oscillating dipoles of all individual molecules in a particle. This is something similar to transmitter and receiver in classical physics. The coupling operator appropriate to this interaction has the form:

$$
\hat{H}_{D}=\sum_{i<j}-\frac{1}{4 \pi \varepsilon_{0}} \vec{\mu}_{i} \cdot \frac{3\left(\vec{\mu}_{j} \cdot \vec{r}_{i j}\right) \cdot \vec{r}_{i j}-\left(\vec{r}_{i j} \cdot \vec{r}_{i j}\right) \cdot \vec{\mu}_{j}}{r_{i j}{ }^{5}}
$$

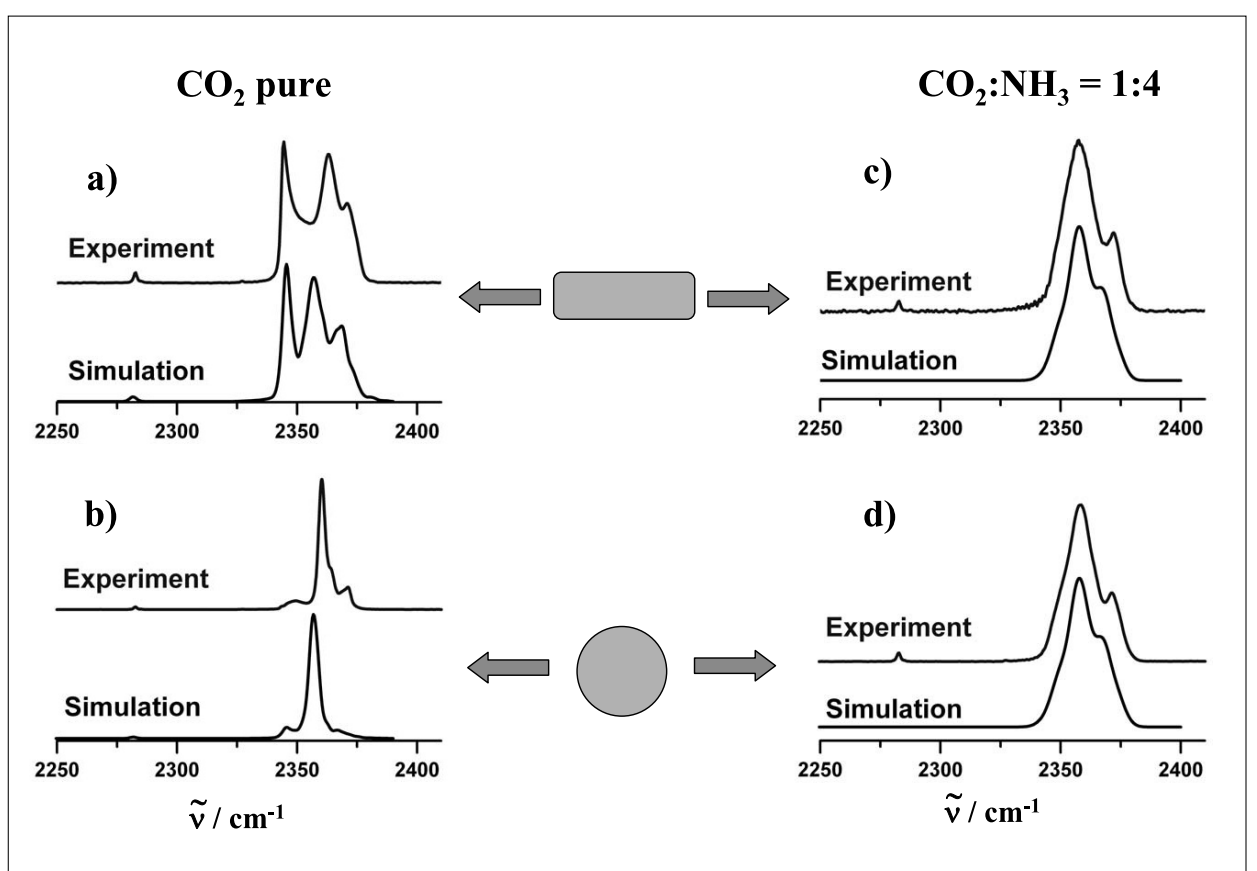

Fig. 1. Infrared spectra in the region of the antisymmetric stretching vibration of $\mathrm{CO}_{2}$ for pure $\mathrm{CO}_{2}$ and mixed $\mathrm{CO}_{2} / \mathrm{NH}_{3}$ particles of different shape. The upper traces show experimental spectra and the lower traces contain the corresponding calculated spectra (see Eqn. (1)). a) For elongated pure $\mathrm{CO}_{2}$ particles; b) For spherical pure $\mathrm{CO}_{2}$ particles; c) For elongated statistically mixed $\mathrm{CO}_{2} / \mathrm{NH}_{3}$ particles; d) For spherical statistically mixed $\mathrm{CO}_{2} / \mathrm{NH}_{3}$ particles. The ratio of $\mathrm{CO}_{2}: \mathrm{NH}_{3}$ amounts to 20:80. The diameter of the particles lies around $50 \mathrm{~nm}$. 
$\vec{\mu}_{i}$ is the dipole moment operator and $r_{i j}$ stands for the distance between the molecules labeled $i$ and $j$. This resonant coupling lifts the degeneracy of the uncoupled states and leads to eigenfunctions that are delocalized over the whole particle. This delocalization explains the distinct shape dependence in the infrared spectra. Calculated spectra for the two different particle shapes discussed above are depicted below the corresponding experimental spectra on the left-hand side in Fig. 1. The comparison with the experiment clearly confirms resonant transition dipole coupling as the microscopic origin of shape effects.

Investigating atmospheric aerosols one is often more interested in the properties of composite than of pure particles. In composite particles, the different chemical substances can be statistically mixed, form inclusions, or build core-shell structures. All these different architectures lead to characteristic patterns in the infrared spectra. The right hand side in Fig. 1 illustrates, as an example, what happens with shape effects in the case of statistically mixed particles. The particles considered here consist of $20 \% \mathrm{CO}_{2}$ and $80 \% \mathrm{NH}_{3}$. Panel c) again shows the infrared spectrum in the region of the antisymmetric stretching vibration of $\mathrm{CO}_{2}$ for predominantly elongated particles and panel d) again depicts the spectrum for mostly spherical particles. The spectra for the two different particle shapes do not differ any longer in the case of statistically mixed particles. This demonstrates that for such mixed particles the information about particle shape is lost in the infrared spectra.

\subsection{Size Effects}

Of course, it is not the particle shape alone which influences the infrared spectra, but also the size of the aggregates [4][11]. Such size effects are particularly important for small particles, which in general means aggregates with radii less than $10 \mathrm{~nm}$. In contrast to large particles, the fraction of molecules which lie in the surface layer is no longer negligible in the case of small particles. It can even outnumber the molecules in the core. Due to the boundary, this surface region has different structural and thus spectroscopic properties than the core of the particles. This is illustrated for ammonia particles in Fig. 2. Panel a) shows the infrared spectrum for ammonia particles with different sizes in the region of the umbrella vibration. With decreasing particle size the band shape becomes broader especially towards higher frequencies. The combination of molecular dynamics simulations with vibrational dynamics calculations shows that this spectral evolution can be understood in terms of surface effects. The surface of the particles has a more amorphous structure than the core of the particles (see Fig. 2b) and thus absorbs in this case at somewhat

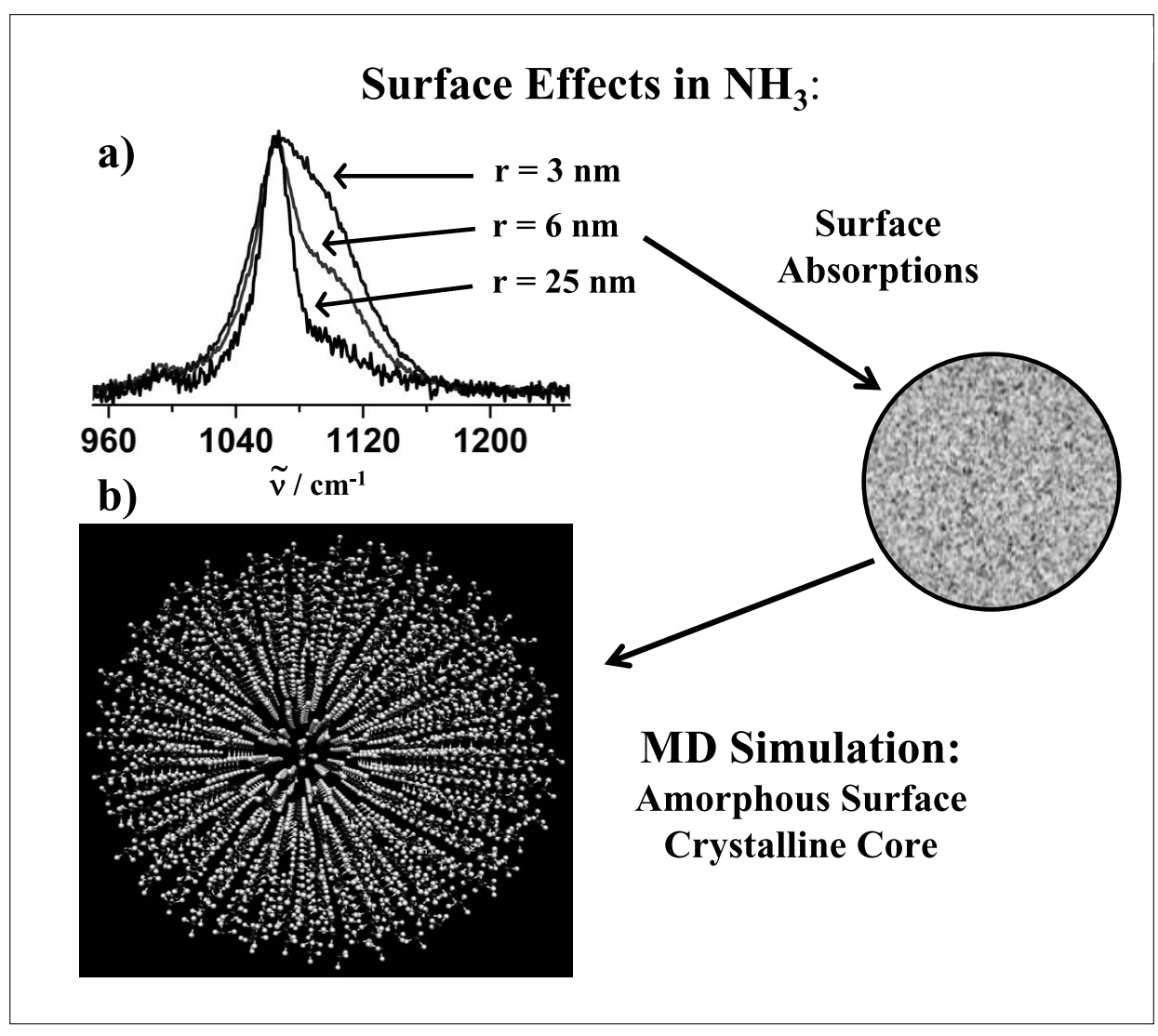

Fig. 2. a) Infrared spectra of ammonia particles for three different particle radii $r$ in the region of the umbrella vibration of ammonia. b) Structure of an ammonia particle with a crystalline core and a partially amorphous surface.

higher energy around $1120 \mathrm{~cm}^{-1}$ (panel a). Fig. 2a illustrates that for smaller particles the relative absorption of the surface increases consistent with a relative increase of surface region in smaller particles.

\section{Reactive Processes}

An important current issue concerns reactive processes involving particles, which includes surface reactions, decay reactions of particles, and particle formation processes starting from gas phase components. Atmospherically relevant chemical processes are, for instance the reaction of ozone with organic acid particles and the formation of sulphuric acid aerosols [20][21] as well as many other complex heterogeneous and homogeneous processes.

The example shown here in Fig. 3 demonstrates that the chemical composition can also depends on the particle preparation method. For that we compare the generation of dihydroxyacetone particles in a collisional cooling cell with the generation in an electrospray [14]. A typical infrared spectrum obtained in the collisional cooling cell is depicted in the lower trace of Fig. 3. As expected one finds the prominent carbonyl stretching band of dihydroxyacetone around $1740 \mathrm{~cm}^{-1}$. A further analysis of the fingerprint region reveals that the mono- mers form hydrogen-bonded chains in the particles as indicated on the right-hand side of Fig. 3b. For the particles generated in the electrospray, by contrast, the carbonyl band is completely missing (Fig. 3a). This tells us that in the electrospray the particle formation is accompanied by a chemical reaction. In the case of dihydroxyacetone this is the chemical dimerization to the cyclic hemiketal (see the right-hand side of Fig. 3a). Under the aqueous conditions of the electrospray this reaction is proton catalyzed whereas the aprotic conditions in the collisional cooling cell inhibit this chemical dimerization.

\section{Drug Particles}

There is also a lot of interest in producing pharmaceutical or cosmetic agents in the form of micro- or nanoparticles [3][12][13][16]. The micronization of pharmaceuticals is a means to increase their bioavailability. One important aspect here is that small particles have a huge surface with correspondingly increased solubility in aqueous solutions even if the bulk substance itself hardly dissolves in water. Another important point is that by choosing the chemical composition particles represent an elegant way for controlled drug delivery. For instance, pH-dependent coat- 


\section{Chemistry with Hydrogen-Bonded Systems: Dihydroxyacetone}

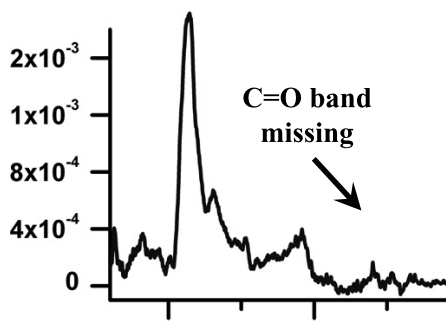

a) Electrospray

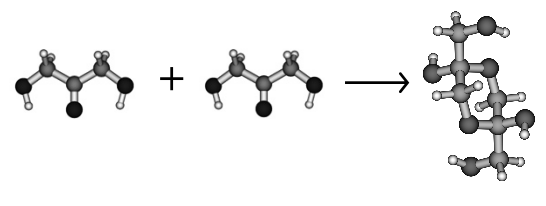

b) Collisional Cooling

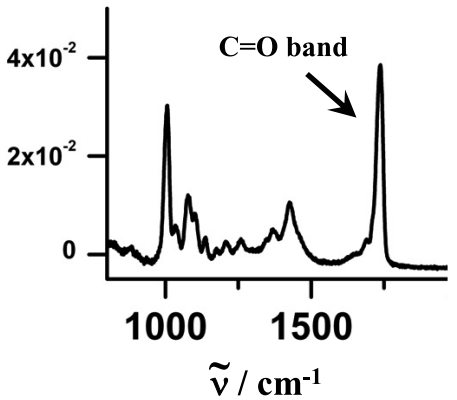

ings can allow for targeted drug release in different intestinal regions.

Some aspects of drug particle formation are summarized in Fig. 4. for the example of phytosterol particles. Phytosterol can be used as an agent to reduce the amount of cholesterol in human blood and as an additive for cosmetics. The particles considered here were generated by rapid expansion of supercritical $\mathrm{CO}_{2}$ solutions. $\mathrm{CO}_{2}$ is a particularly suitable solvent for the formation of thermally labile drug particles since it has low critical data, it is non-toxic, and it can easily be separated from the particulate phase after the particle formation. The picture from scanning electron microscopy (SEM) in Fig. 4 shows that pure phytosterol particles have a strong tendency to coagulate and agglomerate, which leads to a decrease in the active surface of the particles and thus reduces the dissolution rate and the bioavailability. This agglomerated spongy structure consists of primary particles with diameters between 50 $\mathrm{nm}$ and $150 \mathrm{~nm}$. To avoid coagulation and agglomeration, we mix or coat the particles with different biodegradable polymers such as eudragit ${ }^{\circledR}$ or poly-lactic-acid (PLA). We have found that the stabilization of the particles strongly depends on the amount of polymer in the mixture. The beauty of infrared spectroscopy is here that we can determine and thus control the amount of polymer in situ during the particle formation (see infrared spectra in Fig. 4).

\section{Summary}

The purpose of this article was to illustrate how the combination of various particle generation and characterization methods provides a wealth of information about particle properties. An important aspect was the discussion of intrinsic particle properties such as shape and size effects in infrared spectra of these weakly bound molecular aggregates. It has been a central concern to demonstrate that these phenomena can be understood on a microscopic level and that they can even be calculated from molecular properties. On a more practical level, such investigations give insight into atmospherically relevant processes as well as into the course of technically relevant processes of particle formation. This should help us to understand, predict and, in the latter case, optimize them.

\section{Acknowledgements}

Special thanks go to the members of my research group. I am indebted to Prof. M. Suhm for his constant support and encouragement. Financial support was provided by the Deutsche Forschungsgemeinschaft, the German Fonds of the Chemical Industry, and the Natural Sciences and Engineering Research Council of Canada.
Fig. 4. Left: Image from electron microscopy for pure phytosterol particles. The primary particles are strongly coagulated and agglomerated. This can be avoided by mixing/coating the particles with polymers. The amount of polymer can be controlled in situ with infrared spectroscopy (infrared spectra on the right). 
[1] J.H. Seinfeld, S.N. Pandis, 'Atmospheric Chemistry and Physics', Wiley \& Sons, New York, 1998.

[2] P. Ehrenfreund, S.B. Charnley, Annu. Rev. Astron. Astrophys. 2000, 38, 427.

[3] T.L. Rogers, K.P. Johnston, R.O. Williams, Drug Dev. Ind. Pharm. 2001, 27, 1003.

[4] M. Jetzki, A. Bonnamy, R. Signorell, $J$. Chem. Phys. 2004, 120, 11775.

[5] M.K. Kunzmann, R. Signorell, M. Taraschewski, S. Bauerecker, Phys. Chem. Chem. Phys. 2001, 3, 3742.

[6] T.E. Gough, T. Wang, J. Chem. Phys. 1996, 105, 4899.

[7] M.L. Clapp, R.E. Miller, D.R. Worsnop, J. Phys. Chem. 1995, 99, 6317.

[8] R. Disselkamp, G.E. Ewing, J. Chem. Phys. 1993, 99, 2439.

[9] F. Fleyfel, J.P. Devlin, J. Phys. Chem. 1989, 93, 7292.

[10] A. Bonnamy, R. Georges, E. Hugo, R. Signorell, Phys. Chem. Chem. Phys. 2005, 7 , 963.

[11] M.K. Kunzmann, S. Bauerecker, M.A. Suhm, R. Signorell, Spectrochim. Acta A 2003, 59, 2855.

[12] A. Bonnamy, D. Hermsdorf, R. Ueberschaer, R. Signorell, Rev. Sci. Instr. 2005, 76, 053904.
[13] M. Türk, P. Hils, B. Helfgen, K. Schaber, H.-J. Martin, M.A. Wahl, J. Supercrit. Fluids 2002, 22, 75.

[14] M. Jetzki, R. Signorell, J. Chem. Phys. 2002, 117, 8063.

[15] T. Häber, U. Schmitt, M.A. Suhm, Phys. Chem. Chem. Phys. 1999, 1, 5573.

[16] M. Türk, R. Signorell, 'In situ characterization of drug nanoparticles by FTIR spectroscopy', in 'Nanotechnologies for the Life Sciences, Vol. 3 Nanosystem Characterization Tools in the Life Sciences', Ed. C. Kumar, WILEY-VCH, Weinheim, 2005, p: 208-240.

[17] R. Signorell, Mol. Phys. 2003, 101, 3385.

[18] R. Signorell, M.K. Kunzmann, Chem. Phys. Lett. 2003, 371, 260.

[19] R. Signorell, J. Chem. Phys. 2003, 118, 2707.

[20] D.A. Knopf, L.M. Anthony, A.K. Bertram, J. Phys. Chem. A 2005, 109, 5579.

[21] S.B. Couling, J. Fletcher, A.B. Horn, D.A. Newnham, R.A. McPheat, R.G. Williams, Phys. Chem. Chem. Phys. 2003, 5, 4108. 